\title{
- \\ OAB score: A clinical model that predicts the probability of presenting overactive detrusor in the urodynamic study
}

\author{
Leandro Cristian Arribillaga ", Marta Ledesma ${ }^{1}$, Ariel Montedoro ${ }^{1}$, Florencia Pisano 1, Rubén \\ Guillermo Bengió ${ }^{1}$
}

${ }^{1}$ Centro Urológico Profesor Bengió, Córdoba, Argentina

\section{ABSTRACT}

Purpose: To create a predictive model of involuntary detrusor contraction (IDC) to improve the diagnostic accuracy of overactive detrusor (OAD), associating overactive bladder $(\mathrm{OAB})$ symptoms with other clinical parameters in the female population.

Materials and Methods: A total of 727 women were studied retrospectively. In all of them, urodynamic study was conducted for urogynecological causes. Demographics information, personal history, symptoms, physical exam, a 3-day frequency/volume chart and urinary culture, were collected in all patients and they subsequently underwent uroflowmetry and urodynamic studies. A logistic regression model was performed in order to determine independent predictors of presence of IDC. Odd ratio (OR) estimation was used to assign a score to each one of the significant variables $(p \leq 0.05)$ in the logistic regression model. We performed a ROC curve in order to determine the predictive ability of the score in relation to the presence of OAD.

Results: presence of OAD was evident in 210 women (29\%). In the logistic regression analysis, independent predictors of $\mathrm{OAD}$ were urgency, urgency incontinence, nocturia, absence of SUI symptoms, diabetes mellitus, reduction of vaginal trophism and bladder capacity below $150 \mathrm{~mL}$. The probability of IDC diagnosis increases as the score raises (Score 0: 4\% until Score $\geq 10$ : $88 \%$ ). Sensitivity was $71 \%$ and specificity $72 \%$. The area under the curve of $\mathrm{OAB}$ score was $0.784(\mathrm{p}>0.001)$.

Conclusions: $\mathrm{OAB}$ score is a clinical tool that shows higher diagnostic accuracy than $\mathrm{OAB}$ symptoms alone to predict overactive detrusor.

\section{ARTICLE INFO}

Keywords:

Urinary Bladder, Overactive;

Urodynamics

Int Braz J Urol. 2017; 44: 348-54

Submitted for publication:

April 10, 2017

Accepted after revision:

August 20, 2017

Published as Ahead of Print: November 24, 2017

\section{INTRODUCTION}

Overactive Bladder $(\mathrm{OAB})$ is a complex disorder that affects an important group of people, altering their quality of life (1); even to a greater extent than other chronic diseases such as hypertension and diabetes (2). It is a medical entity widely predominant, affecting about $10.7 \%$ of the population worldwide (3).

The consensus of the International Urogynecological Association (IUGA) and the Interna- tional Continence Society (ICS) defines this syndrome as the presence of urgency with or not urge urinary incontinence (UUI), usually associated with an increase of frequency and nocturia in the absence of urinary infection or other underlying pathology (4). This concept implies that the term $O A B$ does not define clearly the etiology. Traditionally, this syndrome has been associated with the presence of involuntary detrusor contractions (IDC) in the filling phase of the cystomanometry; this urodynamic observation is called overactive 
detrusor (OAD). IDC identification in the urodynamic study in patients with $\mathrm{OAB}$ occurs in around $50 \%$ of patients (5), creating controversies among several authors of the initial diagnostic algorithm $(6,7)$. Since the definition of OAB in 2002, there is little evidence that symptoms that belong to this syndrome could diagnose with accuracy the presence of OAD (8). Therefore, the association between $\mathrm{OAB}$ and $\mathrm{OAD}$ is not yet clear. Taking into account the above-mentioned information, the creation of a model that improves the predictive ability of $\mathrm{OAB}$ symptoms by itself is essential; this system should complement them with other clinical variables with the aim of improving the identification of IDC and in this way, reduce the need of unnecessary studies.

The purpose of this study is to create a predictive model of IDC (OAB score) in order to improve the diagnostic accuracy of OAD associating $\mathrm{OAB}$ symptoms with other clinical parameters in the female population.

\section{MATERIALS AND METHODS}

A total of 770 women were studied retrospectively in whom urodynamic study was conducted for urogynecological causes at Centro Urológico Profesor Bengió between January of 2010 and January 2013. Patients with history of urethral stricture, neurological, bladder or colonic disease, pelvic radiotherapy, anticholinergic use and medication that could alter bladder function were excluded from the study.

The study population included 727 female patients. Demographic data was collected from all patients, such as previous surgical procedures and diseases and lower urinary tract symptoms. Subsequently, a complete physical exam was carried out evaluating vaginal trophism, urethral mobility ( $Q$ tip), cough stress test and Valsalva maneuver with full and empty bladder to determine the presence or not of stress urinary incontinence (SUI), and evaluation of the presence of pelvic organ prolapse with empty bladder according to Pelvic Organ Prolapse Quantification system (POP-Q).

In all patients, a 3-day frequency/volume chart was requested and urinary culture should be negative. All urodynamics (UD) were carried out with Ecud Compact ${ }^{\circledR}$ equipment according to ICS protocol of good urodynamic practice (9). Initially, an uroflowmetry was performed with ACE software in a private environment and micturition volume, maximum flow rate $\left(Q_{\max }\right)$ and post void residual urine volume (PVR) measured by catheterization (considered abnormal $>20 \%$ of micturition volume) were recorded. The Multichannel urodynamic study was carried out with the patient in both positions standing up and seated, at an irrigation speed of $30 \mathrm{~mL} / \mathrm{min}$, with water at ambient temperature $\left(25^{\circ} \mathrm{C}\right)$ and filling towards voiding intense desire or $500 \mathrm{~mL}$ irrigation. During the filling cystometry, sensitivity, compliance, bladder capacity and presence of IDC spontaneous or provoked were observed.

Urine incontinence by cough stress tests with Valsalva maneuver every $100 \mathrm{~mL}$ was studied. Lastly, a voiding cystometry (pressure/ flow curve) was carried out.

Patients were divided into two groups according to the presence or not of overactive detrusor. The variables evaluated were age, parity, symptoms such as urgency, UUI, nocturia ( $>$ one night micturition), frequency ( $>7$ daytime micturition), SUI according to IUGA/ICS definitions, voiding alterations: stream, hesitancy and incomplete voiding sensation; prolapse presence (bulk or vaginal weight) and recurrent UTI (+ 2 annual infections), hysterectomy and other pelvic surgeries, menopause. During the physical exam, vaginal trophism, anterior, apical and posterior prolapse were evaluated. The variables studied in uroflowmetry were QMax and PVR. To conclude, bladder capacity was determined according to the frequency/volume chart.

In all patients, using the chi-square statistical method, significant variables $(p \leq 0.05)$ for $\mathrm{OAD}$ were entered in a logistics regression model in order to determine independent predictors for the presence of involuntary detrusor contractions. OR unity value was used to assign a score to each one of the significant variables $(p \leq 0.05)$ in the regression logistics model. The sum of these in each patient determines a score (varying from 0 to 10). In the end, we performed a ROC curve in order to evaluate the ideal score cut-off point, determining its sensitivity and 
specificity; the predictive capacity of the score in relation to the $\mathrm{OAD}$ presence was determined by the area below the curve.

\section{RESULTS}

The mean age of the studied population was 60.5 years old (23-89). The presence of OAD was demonstrated in 210 women (29\%). Other diagnostic findings were urodynamic SUI in 74\% of the cases, prolapse 35\%, voiding alterations in $25 \%$ and $10 \%$ of UTI recurrence. In Table- 1 we demonstrate the clinical and urodynamic comparison between patients with and without OAD.

In the univariate analysis, we could demonstrate that factors that predispose the presence of OAD were: age $>60$ years old, urgency, UUI, nocturia, frequency, UTI recurrence, absence of SUI symptoms, diabetes, reduction of vaginal trophism, POP posterior and bladder capacity $<150$ $\mathrm{mL}$ in the frequency/volume chart. These variables were included in the multivariate model observed in Table-2.

In the logistics regression analysis, we observed that independent predictors for $\mathrm{OAD}$ are urgency, UUI, nocturia, absence of SUI symptoms, presence of diabetes, reduction of vaginal trophism and bladder capacity below $150 \mathrm{~mL}$. In Table- 3 we assigned the result of the score in relation to $\mathrm{OR}$.

Table-4 presents the $\mathrm{OAB}$ score, where for each result obtained by the sum of parameters in each patient, it is stablished the possibility of presenting $\mathrm{OAD}$.

As we have demonstrated, as the score increases, the probability for presenting IDC in the urodynamic study increases as well starting at $4 \%$ with Score 0 , reaching $88 \%$ with Score $\geq 10$. If we only include the presence of symptoms of $\mathrm{OAB}$ (urgency, UUI, nocturia and frequency). the sensitivity is $57.4 \%$, the specificity $83.7 \%$ and the area under the curve 0.70. After the ROC curve was drawn we determined that the ideal cut-off point of the Score is 5, with a sensitivity of $71 \%$ and specificity of $72 \%$. In Figure-1 we point out that the area under the curve in order to determine the predictive capacity of the OAB score is $0.784(\mathrm{p}<0.001)$.

\section{DISCUSSION}

Overactive bladder syndrome can affect significantly quality of life. Therefore, its etiology and an accurate clinical diagnosis have been important goals over the time. The exact definition for ICS refers to a syndrome where the principal symptom is urgency, with or without UUI, usually associated to frequency and nocturia. Although according to the recommendation of the International societies (ICS/IUGA), the urodynamic study is not routinely necessary in patients with $\mathrm{OAB}$, the cystometric evaluation, as part of the clinical and diagnostic evaluation, is widely accepted as the gold standard in the evaluation of low urinary tract dysfunctions, including OAD (10); the diagnosis of OAD is based on the presence of involuntary detrusor contractions in the filling and storage phases in women with $\mathrm{OAB}$ symptoms $(4$, 8). Thus, many professionals believe that a correct diagnosis cannot be determined in some patients if urodynamic is not carried out and therefore will not receive a proper treatment, considering that the bladder has been described as an unreliable witness (11). Nevertheless, the reproduction of OAB symptoms with IDC presence in the urodynamic study is not yet clear. In our population with urgency, the presence of $\mathrm{OAD}$ is $35 \%$ and it can rise to $57 \%$ if we add other $\mathrm{OAB}$ symptoms (UUI, nocturia and frequency). Several previous series have documented an incidence of OAD that ranges between $32-54 \%$ in women with $\mathrm{OAB}(5,7,12$, 13). At the same time, the routine use of ambulatory urodynamic study raises the diagnosis to 70\% (14); although it is also important to point out the presence of OAD in $68 \%$ of voluntary asymptomatic women, limiting its use as a first line practice (15). However, these variations can be subject to different factors that may affect the detection of $\mathrm{OAD}$, including speed in filling, position of the patient and provocation maneuvers (16). This shows the need of a more accurate clinical model to reduce unnecessary urodynamic investigations from the diagnostic point of view with higher morbidity (invasiveness).

Colli et al. carried out a revision of 20 studies (1980-2000), evaluating sensitivity and specificity of $\mathrm{OAD}$ in patients with $\mathrm{OAB}$ symptoms, 
Table 1 - Comparison of clinical and urodynamic profile of patients with and without OAD.

\begin{tabular}{|c|c|c|c|c|}
\hline Variable & No OAD (n:517) & OAD (n:210) & $\mathrm{OR}$ & $P$ \\
\hline Age $>60$ year old & $270(52.2 \%)$ & $129(61.4 \%)$ & 1.46 & 0.02 \\
\hline Parity $\leq 1$ & $114(22.1 \%)$ & $52(24.8 \%)$ & 1.16 & 0.42 \\
\hline Parity $\geq 2$ & $403(77.9 \%)$ & $158(75.2 \%)$ & 1.21 & 0.43 \\
\hline \multicolumn{5}{|l|}{ Symptoms } \\
\hline Urgency & $321(62.1 \%)$ & $185(88.1 \%)$ & 4.5 & $<0.001$ \\
\hline UUI & $249(48.1 \%)$ & $167(79.5 \%)$ & 4.2 & $<0.001$ \\
\hline Nocturia & $126(24.4 \%)$ & $141(67.2 \%)$ & 6.3 & $<0.001$ \\
\hline Frequency & $80(15.5 \%)$ & $93(44.3 \%)$ & 4.3 & $<0.001$ \\
\hline Voiding alterations & $121(23.4 \%)$ & $58(27.6 \%)$ & 1.25 & 0.23 \\
\hline POP & $189(36.5 \%)$ & $69(32.9 \%)$ & 0.85 & 0.34 \\
\hline UTI recurrence & $46(8.9 \%)$ & $29(13.8 \%)$ & 1.64 & 0.04 \\
\hline Absence of SUI & $107(20.7 \%)$ & $85(40.5 \%)$ & 2.6 & $<0.001$ \\
\hline \multicolumn{5}{|l|}{ Background } \\
\hline Hysterectomy & $129(24.9 \%)$ & $58(27.6 \%)$ & 1.15 & 0.45 \\
\hline Pelvic Surgery & $68(13.1 \%)$ & $30(14.3 \%)$ & 1.10 & 0.68 \\
\hline Menopause & $396(76.6 \%)$ & $171(81.4 \%)$ & 1.34 & 0.15 \\
\hline Diabetes & $21(4 \%)$ & $19(9.1 \%)$ & 2.35 & 0.007 \\
\hline \multicolumn{5}{|l|}{ Physical exam } \\
\hline Vaginal Trophism Reduction & $215(41.6 \%)$ & $120(57.1 \%)$ & 1.87 & 0.001 \\
\hline Presence of SUI & $205(39.6 \%)$ & $66(31.4 \%)$ & 1.43 & 0.05 \\
\hline Anterior POP & $169(39.7 \%)$ & $59(28.1 \%)$ & 0.80 & 0.22 \\
\hline Middle POP & $76(14.7 \%)$ & $40(19.1 \%)$ & 1.37 & 0.14 \\
\hline Posterior POP & $85(16.4 \%)$ & $49(23.3 \%)$ & 1.55 & 0.02 \\
\hline $\begin{array}{l}\text { Bladder capacity }<150 \mathrm{~mL} \text { in frequency/volume } \\
\text { chart }\end{array}$ & $60(11.6 \%)$ & $57(27.1 \%)$ & 2.84 & $<0.001$ \\
\hline \multicolumn{5}{|l|}{ Uroflowmetry } \\
\hline$Q_{\max }<20 \mathrm{~mL} / \mathrm{sec}$ & $67(12.9 \%)$ & $30(14.3 \%)$ & 1.12 & 0.63 \\
\hline High PVR & $45(8.7 \%)$ & $24(11.4 \%)$ & 1.35 & 0.25 \\
\hline
\end{tabular}


Table 2 - Multivariate analysis of OAD clinical predictors.

\begin{tabular}{lccc}
\hline Variable & OR & Cl 95\% & P \\
\hline Age $>60$ years old & 0.73 & $0.46-1.17$ & 0.19 \\
Urgency & 1.56 & $1.04-2.66$ & $\mathbf{0 . 0 5}$ \\
UUI & 1.97 & $1.08-3.60$ & $\mathbf{0 . 0 2}$ \\
Nocturia & 3.46 & $2.21-5.42$ & $<\mathbf{0 . 0 0 1}$ \\
Frequency & 1.33 & $0.83-2.13$ & 0.24 \\
UTI recurrence & 1.09 & $0.61-1.93$ & 0.77 \\
Absence of SUI symptoms & 2.33 & $1.50-3.64$ & $<\mathbf{0 . 0 0 1}$ \\
Diabetes & 2.53 & $1.20-5.33$ & $\mathbf{0 . 0 1}$ \\
Vaginal Trophism reduction & 1.59 & $1.01-2.38$ & $\mathbf{0 . 0 5}$ \\
POP posterior & 1.45 & 0.91 .2 .29 & 0.11 \\
bladder capacity $<150 \mathrm{~mL}$ & 2.12 & $1.20-3.08$ & $\mathbf{0 . 0 0 6}$ \\
\hline
\end{tabular}

Table 3 - Variable score according to OR logistics regression analysis.

\begin{tabular}{lc}
\hline Variable & Point Score \\
\hline Nocturia & $\mathbf{3}$ \\
Vesical capacity $<150 \mathrm{~mL}$ & 2 \\
Absence of SUI symptoms & 2 \\
Diabetes & 2 \\
Urgency & 1 \\
UUI & 1 \\
Vaginal Trophism reduction & 1 \\
\hline
\end{tabular}

Table 4 - OAB Score and IDC probability in the urodynamic study.

\begin{tabular}{lc}
\hline Score & IDC Probability \\
\hline 0 & $4 \%$ \\
1 & $8 \%$ \\
2 & $20 \%$ \\
3 & $16 \%$ \\
4 & $24 \%$ \\
5 & $29 \%$ \\
6 & $45 \%$ \\
7 & $61 \%$ \\
8 & $65 \%$ \\
9 & $86 \%$ \\
$\geq 10$ & $88 \%$ \\
\hline
\end{tabular}

Figure 1 - OAD and OAB Score ROC curve.

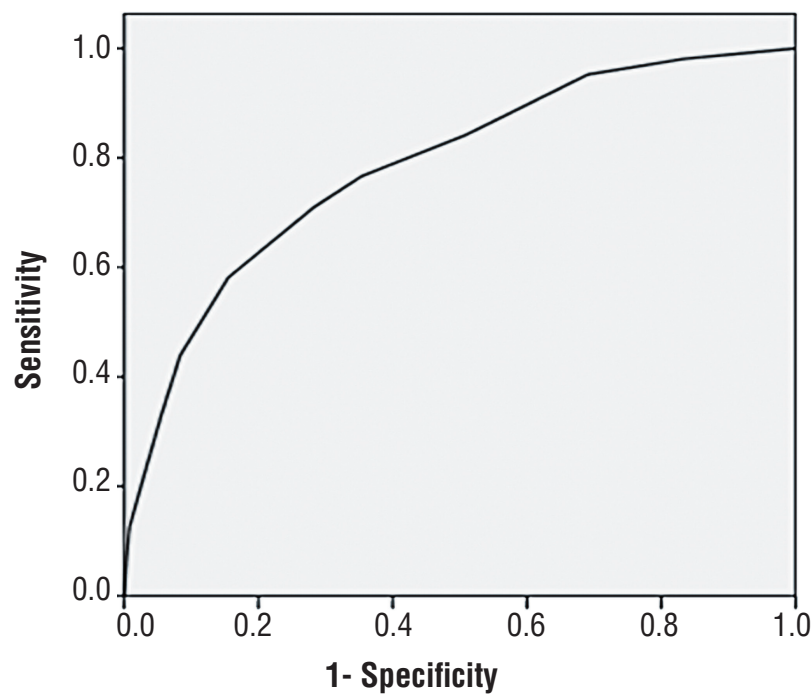

finding a sensitivity of $0.69(0.36-0.96)$ and a specificity of $0.60(0.21-0.97)$ to predict IDC presence based on symptoms (17). This data is in accordance to our series.

In order to improve the ability to predict DHA along with symptoms of OAB (except urinary frequency), other clinical variables were analyzed by a multivariate model of logistics regression (bladder function capacity $<150 \mathrm{~mL}$ in 3 day frequency/volume chart, absence of SUI symptoms, diabetes and vaginal trophism reduction). Urinary frequency was excluded since it 
was not an independent predictor of OAD, similarly to previous studies, without statistical signifance $(7,18,19)$.

$\mathrm{OAB}$ Score was proposed to improve the diagnostic capacity in patients with only $\mathrm{OAB}$ symptoms (sensitivity $71 \%$, specificity $72 \%$ and ROC curve 0.78), perhaps creating a clinical tool that predicts more accurately the presence of IDC.

Some previous observations have included other signs and symptom to improve predictability of $\operatorname{OAD}(5,15)$. In our studied population, we found light differences in the age of patient with or without $\mathrm{OAD}$, although it was not expressed as an independent predictor as in previous reports, where it was observed 3-5 years of differences in patients with presence of IDC $(13,20)$. Harris and Haylen demonstrated that nulliparity increased the risk of IDC, which was not concurrent to our findings $(18,21)$. In the same manner that our model series, it was previously demonstrated that the absence of SUI has a high possibility of OAD (18, 22). Currently, there are controversies on the risk of $\mathrm{OAD}$ in relation to POP presence, hysterectomy and previous pelvic surgery, although in our series this was not demonstrated as a variable to be considered statistically significant $(12,23)$.

Previous studies did not demonstrate variable differences in daytime or nocturnal frequency in the frequency/volume chart; however, women with IDC presence have a lower functional capacity in some preceding series $(12,13)$.

Diabetes mellitus (DM) is another independent risk factor in our series. Epidemiological study has shown that DM is an independent factor for $\mathrm{OAB}$ (24).

Just as in our study, several authors have shown that vaginal atrophy due to estrogen insufficiency is a recognized cause or contributory factor in overactive bladder $(25,26)$.

Two previous publications have tried to improve clinical diagnosis of $\mathrm{OAD}$ by predicting models. Vella et al. described a Score after a clinical survey of 171 women that included 9 symptoms, improving the sensitivity and specificity; yet it is not clear whether their selected study model was univariate or multivariate (27). Haylen et al. improves the prediction of OAD adding to the classical $\mathrm{OAB}$ symptoms (except urinary fre- quency), the lower presence of parity (0-1), absence of SUI and absence of POP signs, though such improvement was minimum and would not be of great clinical utility (ROC curve 0.70 vs. 0.74 ) (18).

In our study, the proposal of an OAB Score was based in the construction of a clinical model that generated more accuracy in OAD diagnosis, given that if this diagnosis is inappropriate, it can lead to bad medical treatments, while the possibility of relieving the patient from its symptoms is less probable. Also, it could reduce the need of urodynamic studies and introduce rapidly first line therapies in $\mathrm{OAB}$, particularly conservative behavioral measures and medications. Therefore, our score could be very useful in primary practice when managing patients with $\mathrm{OAB}$, since it can be reproduced easily, improve cost-effectiveness and reduce the use of unnecessary treatment that generate disappointment to the patient, with higher morbidity and adverse events that worsen even more quality of life.

\section{CONCLUSIONS}

$\mathrm{OAB}$ score is a clinical tool with higher diagnostic precision than $\mathrm{OAB}$ symptoms alone to predict OAD. It is easily reproducible as is based on clinical parameters commonly used in the daily urogynecological practice.

\section{CONFLICT OF INTEREST}

None declared.

\section{REFERENCES}

1. Coyne KS, Sexton CC, Irwin DE, Kopp ZS, Kelleher CJ, Milsom I. The impact of overactive bladder, incontinence and other lower urinary tract symptoms on quality of life, work productivity, sexuality and emotional well-being in men and women: results from the EPIC study. BJU Int. 2008;101:1388-95.

2. Litman HJ, McKinlay JB. The future magnitude of urological symptoms in the USA: projections using the Boston Area Community Health survey. BJU Int. 2007;100:820-5. Erratum in: BJU Int. 2007;100:971.

3. Kelleher CJ, Cardozo LD, Khullar V, Salvatore S. A new questionnaire to assess the quality of life of urinary incontinent women. Br J Obstet Gynaecol. 1997;104:1374-9. 
4. Haylen BT, de Ridder D, Freeman RM, Swift SE, Berghmans $B$, Lee J, et al. An International Urogynecological Association (IUGA)/International Continence Society (ICS) joint report on the terminology for female pelvic floor dysfunction. Neurourol Urodyn. 2010;29:4-20.

5. Digesu GA, Khullar V, Cardozo L, Salvatore S. Overactive bladder symptoms: do we need urodynamics? Neurourol Urodyn. 2003;22:105-8. Erratum in: Neurourol Urodyn. 2003;22:356.

6. Blaivas $\mathrm{JG}$. The bladder is an unreliable witness. Neurourol Urodyn. 1996;15:443-5.

7. Hashim H, Abrams P. Is the bladder a reliable witness for predicting detrusor overactivity? J Urol. 2006;175:191-4; discussion 194-5.

8. Abrams P, Cardozo L, Fall M, Griffiths D, Rosier P, Ulmsten $\mathrm{U}$, et al. Standardisation Sub-committee of the International Continence Society. The standardisation of terminology of lower urinary tract function: report from the Standardisation Sub-committee of the International Continence Society. Neurourol Urodyn. 2002;21:167-78.

9. Schäfer W, Abrams P, Liao L, Mattiasson A, Pesce F, Spangberg A, et al. International Continence Society. Good urodynamic practices: uroflowmetry, filling cystometry, and pressure-flow studies. Neurourol Urodyn. 2002;21:261-74.

10. Matharu G, Donaldson MM, McGrother CW, Matthews RJ. Relationship between urinary symptoms reported in a postal questionnaire and urodynamic diagnosis. Neurourol Urodyn. 2005;24:100-5.

11. Flisser AJ, Blaivas JG. Role of cystometry in evaluating patients with overactive bladder. Urology. 2002;60(5 Suppl 1):33-42; discussion 42.

12. Giarenis I, Mastoroudes H, Srikrishna S, Robinson D, Cardozo $\mathrm{L}$. Is there a difference between women with or without detrusor overactivity complaining of symptoms of overactive bladder? BJU Int. 2013;112:501-7.

13. Jeong SJ, Lee SC, Jeong CW, Hong SK, Byun SS, Lee SE. Clinical and urodynamic differences among women with overactive bladder according to the presence of detrusor overactivity. Int Urogynecol J. 2013;24:255-61.

14. Radley SC, Rosario DJ, Chapple CR, Farkas AG. Conventional and ambulatory urodynamic findings in women with symptoms suggestive of bladder overactivity. J Urol. 2001;166:2253-8.

15. Heslington K, Hilton P. Ambulatory monitoring and conventional cystometry in asymptomatic female volunteers. Br J Obstet Gynaecol. 1996;103:434-41.

16. Hosker G, Rosier P, Gajewski J, Sand P, Szabo L, Capewell A. Dynamic testing. In Abrams P, Cardozo L, Khoury S, Wein A eds, Incontinence, 4th International Consultation on Incontinence, 4th edn. Paris: Health Publications Ltd, 2009: 413-522.
17. Colli E, Artibani W, Goka J, Parazzini F, Wein AJ. Are urodynamic tests useful tools for the initial conservative management of non-neurogenic urinary incontinence? A review of the literature. Eur Urol. 2003;43:63-9.

18. Haylen BT, Chiu TL, Avery D, Zhou J, Law M. Improving the clinical prediction of detrusor overactivity by utilizing additional symptoms and signs to overactive bladder symptoms alone. Int Urogynecol J. 2014;25:1115-20.

19. Cantor TJ, Bates CP. A comparative study of symptoms and objective urodynamic findings in 214 incontinent women. $\mathrm{Br}$ J Obstet Gynaecol. 1980;87:889-92.

20. Haylen BT, Chetty N, Logan V, Schulz S, Verity L, Law M, et al. Is sensory urgency part of the same spectrum of bladder dysfunction as detrusor overactivity? Int Urogynecol J Pelvic Floor Dysfunct. 2007;18:123-8.

21. Harris RL, Cundiff GW, Coates KW, Bump RC. Urinary incontinence and pelvic organ prolapse in nulliparous women. Obstet Gynecol. 1998;92:951-4.

22. Lagro-Janssen AL, Debruyne FM, van Weel C. Value of the patient's case history in diagnosing urinary incontinence in general practice. Br J Urol. 1991;67:569-72.

23. de Boer TA, Slieker-ten Hove MC, Burger CW, Vierhout ME. The prevalence and risk factors of overactive bladder symptoms and its relation to pelvic organ prolapse symptoms in a general female population. Int Urogynecol J. 2011;22:569-75.

24. Lawrence JM, Lukacz ES, Liu IL, Nager CW, Luber KM. Pelvic floor disorders, diabetes, and obesity in women: findings from the Kaiser Permanente Continence Associated Risk Epidemiology Study. Diabetes Care. 2007;30:2536-41.

25. Banakhar MA, Al-Shaiji TF, Hassouna MM. Pathophysiology of overactive bladder. Int Urogynecol J. 2012;23:975-82.

26. Toozs-Hobson P. The overactive bladder.Obstetrics, Gynaecology and Reproductive Medicine 2010; 20: 300-5.

27. Vella M, Robinson D, Cardozo L, Srikrishna S, Cartwright R. Predicting detrusor overactivity using a physician-based scoring system. Int Urogynecol J Pelvic Floor Dysfunct. 2008;19:1223-7. 